\title{
Do Very Short Gamma Ray Bursts Originate from Primordial Black Holes? Review
}

\author{
David B. Cline ${ }^{1}$, Stanislaw Otwinowski ${ }^{1}$, Bozena Czerny ${ }^{2}$, Agnieszka Janiuk ${ }^{3}$ \\ ${ }^{1}$ Department of Physics and Astronomy, University of California, Los Angeles, USA \\ ${ }^{2}$ Nicolaus Copernicus Astronomical Center, Warsaw, Poland \\ ${ }^{3}$ Center for Theoretical Physics Polish Academy of Sciences, Warsaw, Poland \\ E-mail:dcline@physics.ucla.edu,stanislaw.otwinowski@cern.ch,\{bcz,agnes\}@camk.edu.pl \\ Received April 4, 2011; revised June 3, 2011; accepted June 17, 2011
}

\begin{abstract}
We present the state of current research of Very Short Gamma Ray Bursts (VSGRBs) from seven GRB detectors. We found that VSGRBs form distinct class of GRBs, which in our opinion, in most cases can originate from the evaporating Primordial Black Holes (PBHs). Arguments supporting our opinion: 1) GRBs with time duration $\left(T_{90}\right)<100 \mathrm{~ms}$ form distinct class: VSGRBs. 2) We observe significant anisotropy in the galactic angular distribution of BATSE VSGRB events. 3) V/Vmax distribution for BATSE VSGRB events indicates the local distance production. 4) VSGBBs have more energetic $\gamma$-ray burst than other GRBs with longer duration (KONUS). 5) We observe small number of afterglows in SWIFT VSGRB sample (25\%), in contrast with the noticeable afterglow frequency in SGRB sample (78\%). 6) Time profile of rising part BATSE VSGRBs is in agreement with the evaporation PBH model.
\end{abstract}

Keywords: Black Hole Physics, Cosmology, Gamma Ray Burst, Primordial Black Hole

\section{Introduction}

Very shortly after the Big Bang, pressure and temperature were extremely great. Under these conditions, simple fluctuation in the density of the matter could have resulted in creation of black holes. Primordial black holes (PBHs) would persist to the present [1-3]. Hawking [1] showed that PBHs of mass less than $\sim 5 \cdot 10^{14} \mathrm{~g}$ must have been evaporated by now.

One way to detect PBHs is by their Hawking radiation, when they evaporate ("explode") now. The properties of PBH burst emission are model dependent and were estimated [4] for $\gamma$-ray burst in the range of tens of ms with luminosity $\sim 10^{33} \mathrm{erg}$. A first-order phase transition was assumed and there is now evidence that the Quark-Gluon phase phase transition is at least part first order. If we supposed the phase transition in final state of evaporation [4] - this means "explosion" with upper limit of particles ' and $\gamma$ ' energy, then we should expect modification of the final state of Hawking evaporation process. Because we expect a fireball to form low-energy photons will be emitted with a very short pulse.

\section{Time Duration}

\section{BATSE}

For the GRB from the detector BATSE we divide into three classes according to their time duration $\left(\mathrm{T}_{90}\right)$ : Long Gamma Ray Bursts (LGRBs) $\left(\mathrm{T}_{90}>1\right.$ or $\left.2 \mathrm{~s}\right)$; Short Gamma Ray Bursts (SGRBs) (1 or $2 \mathrm{~s}>\mathrm{T}_{90}>0.1 \mathrm{~s}$ ); and VSGRBs $\left(T_{90} \leq 0.1 \mathrm{~s}\right)$. See Figure 1. We assume that the VSGRBs constitute a separate class of GRBs with log normal duration distribution and we fit the time distribution in Figure 1 with a three-population model. The fit is excellent but does not give significant evidence for a three-population model. The arrow $[\downarrow]$ shows position of VSGRBs.

In Figure 1 and Figure 2 we present GRB duration distribution for 7 detectors. The distributions are different and they depend on different sensitivity (detector dimension), detected spectrum energy, the background dependence, trigger time length and other detector characteristics. There are well visible noticeable two or three groups: long and short time bursts, there is also possible to distinguish middle time bursts (energy extended). Looking at the shortest burst we can see some tail for each detector, which sticking out of the log normal fitted 
BATSE GRB EVENTS (SINCE APRIL 21,1991 TILL NOVEMBER 19,1990)

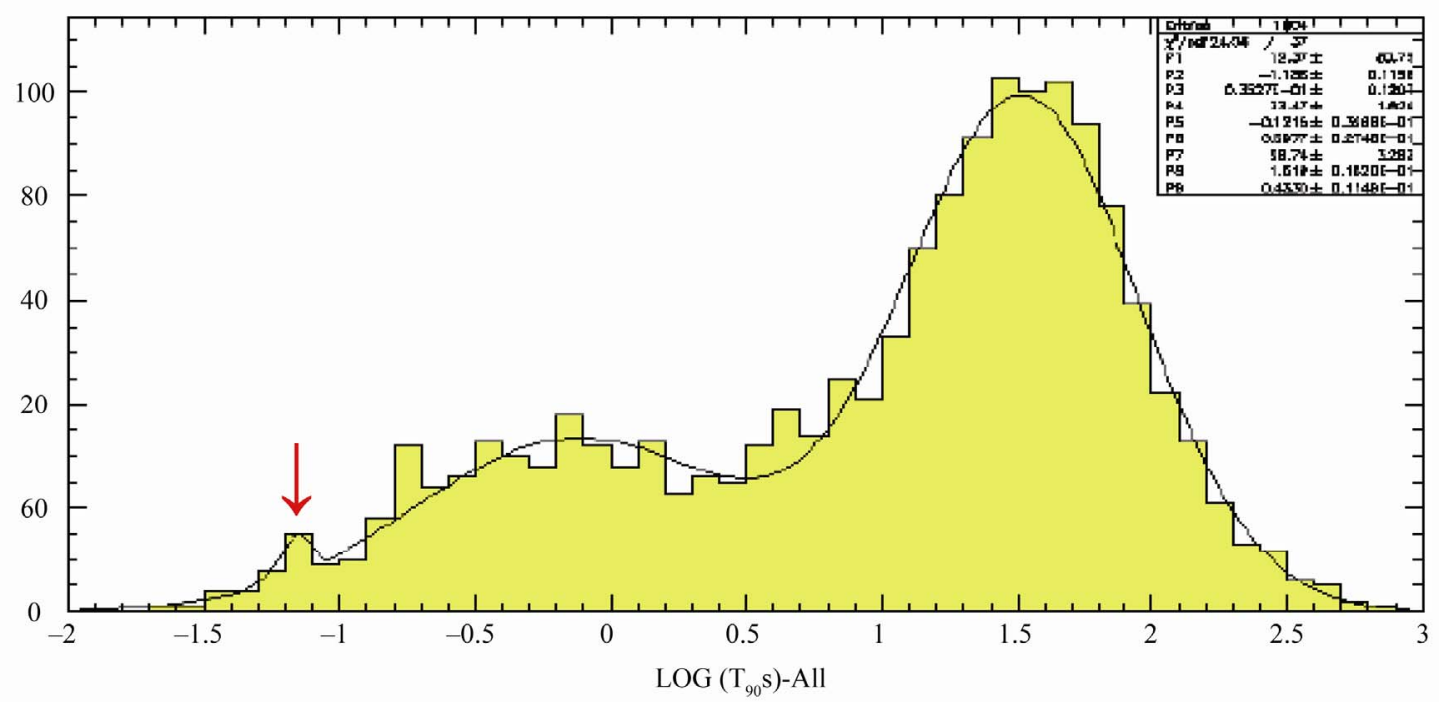

Figure 1. Duration distribution, $\mathrm{T}_{\mathbf{9 0}}$ for all GRB from BATSE detector.

curves. That is not statistically significant but it is observed for every detector even if trigger minimum length reduces probability detection of shortest burst! In total it seems to be statistically significant. Additionally the energetic analysis of KONUS short and very short bursts mark out VSGRB group-see Figure 8. We note that there is a correlation between the relative excess of the short events number and the detector energy range. In particular, Integral data are gathered in the range $20 \mathrm{keV}$ - $8 \mathrm{MeV}$ and the short events sample is most numerous. The hardest bursts are observed by Fermi $(8 \mathrm{keV}-30$ $\mathrm{MeV}$ ) and in this case two groups of short events can be seen, however the statistics for this instrument is still quite poor. Also the non isotropic events show excess for GRBs with duration shorter than $0.1 \mathrm{~s}$, look at Figure 4. All this suggests that we really observe distinct group of VSGRBs with $\mathrm{T}_{90} \leq 0.1 \mathrm{~s}$.

\section{Anisotropy}

The angular distribution of VSGRB and SGRB in Galactic Coordinates is shown in Figure 3. The sky was divided into 8 equal regions. In the case of isotropic distribution the number of bursts in each region should be described by the Poisson distribution. The histograms of such values are shown in Figure 3. The probability of any number of bursts in a single zone multiplied by the number of zones (8) is shown with full circles. For $448 \mathrm{SB}\left(0.1 \mathrm{~s}<\mathrm{T}_{90}<2 \mathrm{~s}\right)$ we see conformity with isotropic distribution, but for VSB $\left(\mathrm{T}_{90} \leq 0.1 \mathrm{~s}\right)$ the observed distribution is strongly improbable to be isotropic. The number of bursts in one of the regions (roughly in the direction of the Galactic Anticenter) is 20 , which is much higher than the expected average of 51/8. The probability to find twenty or more events (from the total number of fifty one) in the region of $1 / 8$ area is 0.00007 . This result argues for other explanation than the statistical fluctuation. Background in the direction of the Galactic Center is 12,500 \pm 1000 counts $\cdot \mathrm{s}^{-1}$, while the mean level of the background outside this region is $13,800 \pm 1300$ counts $\cdot \mathrm{s}^{-1}$, and the total number of SB in these regions is slightly lower than the expected average (but within the expected error). Therefore, background anisotropy cannot be responsible for the observed distribution of the VSB across the sky [5].

In Figure 4 we show the distribution of the number of the GRBs excess as a function of $T_{90}$. The excess from the isotropic distribution is calculated as a difference between the GRBs number in the chosen region and the sum of all GRBs number in other regions divided by seven. This study shows that the incompatibility with isotropic distribution is seen only for GRBs with $\mathrm{T}_{90} \leq 0.1 \mathrm{~s}$. We observe the total excess of $15.71 \pm 4.52$ bursts in this region [5].

The BATSE VSGRB angular Galactic coordinate distribution was reanalyzed using factorial moments and cumulants analysis [6]. Authors present VSGRB angular distribution with very suggestive method, which consists in presenting GRB coordinate point to $10^{\circ}, 25^{\circ}$ and $40^{\circ}$ radius cone, see Figure 5. The detailed first four factorial moments analysis gives as a result the probability < $3 \cdot 10^{-5}$ for the chance of such fluctuation from uniform distribution. This is in agreement with our earlier, simple estimation: $7 \cdot 10^{-5}$, see Figure 3, VSB-b. It means the effect itself is on about $4 \sigma$ level. The analyses in Figures 3-5 are all independent.

The authors analyze, with cumulants method, up to $5^{\text {th }}$ 

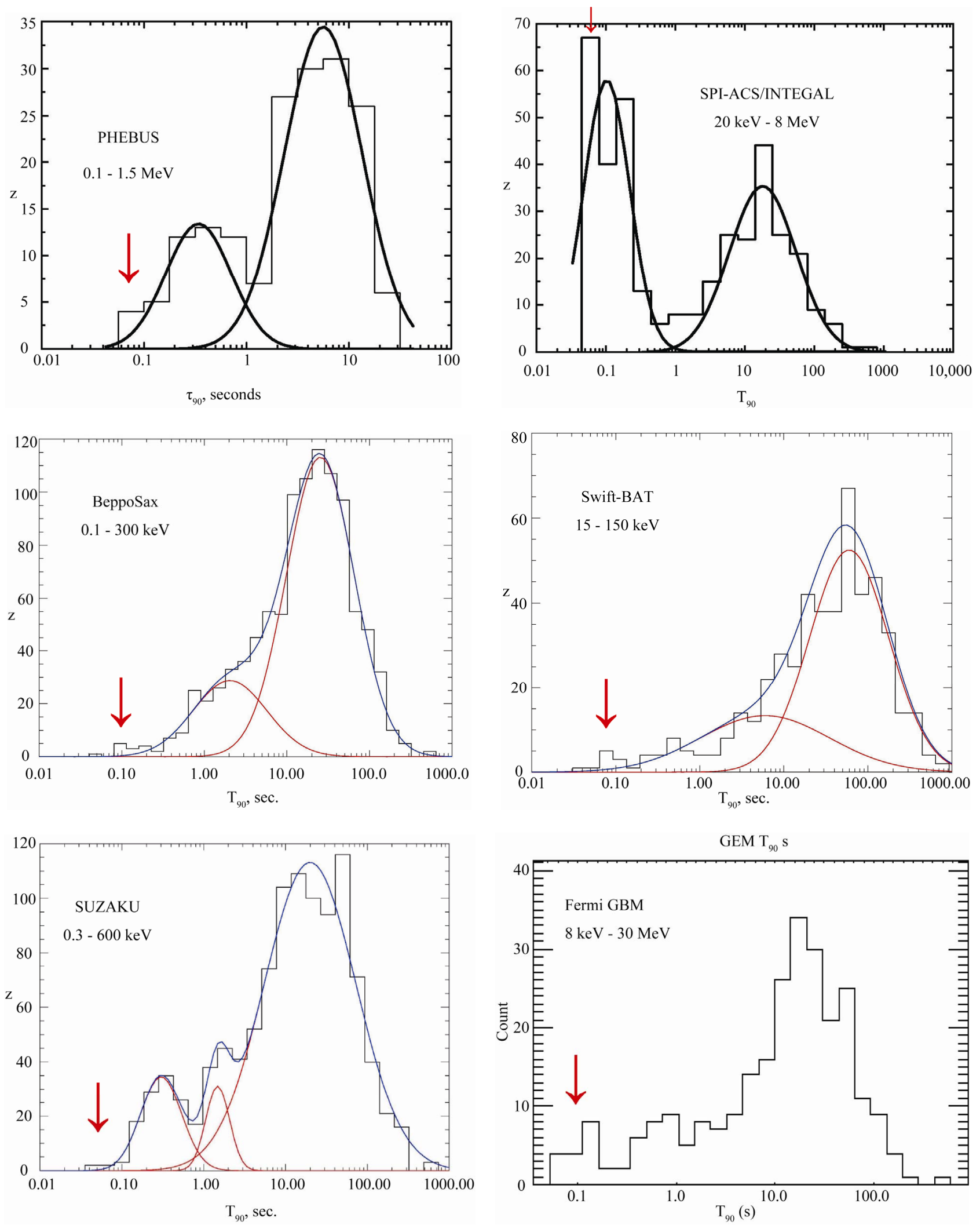

Figure 2. Duration distribution, T $_{90}$, for GRBs from PHEBUS, SPI-ACS/INTEGRAL, BeppoSax, Swift and SUZAKU detectors according to [8] and Fermi GBM GRB catalog. The arrow $[\downarrow]$ shows position of VSGRBs. Energy range is shown for each detector below its name. 
BATSE GRB EVENTS (21/04/1991- 26/05/2000)

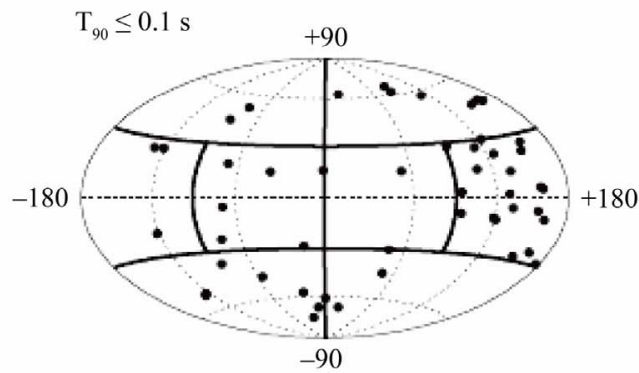

Galactic Coordinates- 8 regions

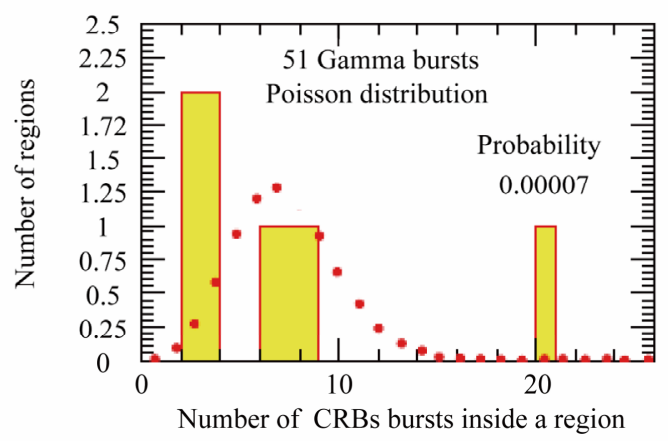

VSB-b)

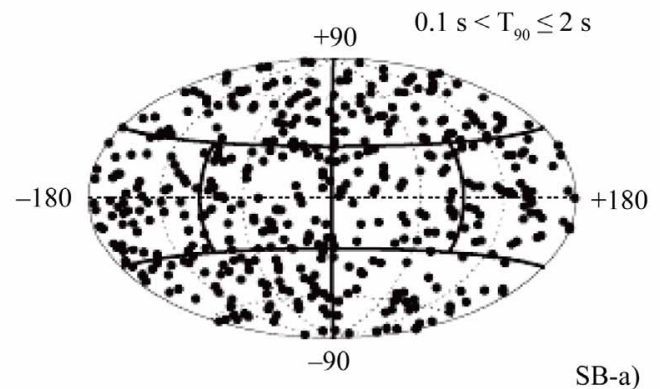

Galactic Coordinates- 8 regions

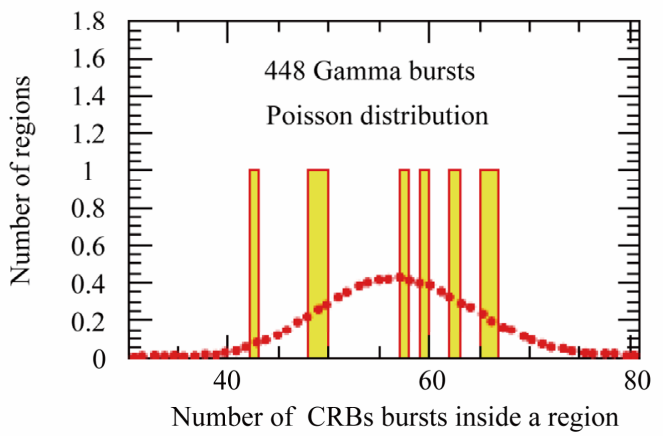

SB-b)

Figure 3. Angular distribution of the GRB in Galactic Coordinates and the corresponding histograms, in comparison with Poisson distribution predictions for Very Short Burst (VSB) and Short Burst (SB) $\mathbf{T}_{90}$ ranges (full circles) [5].

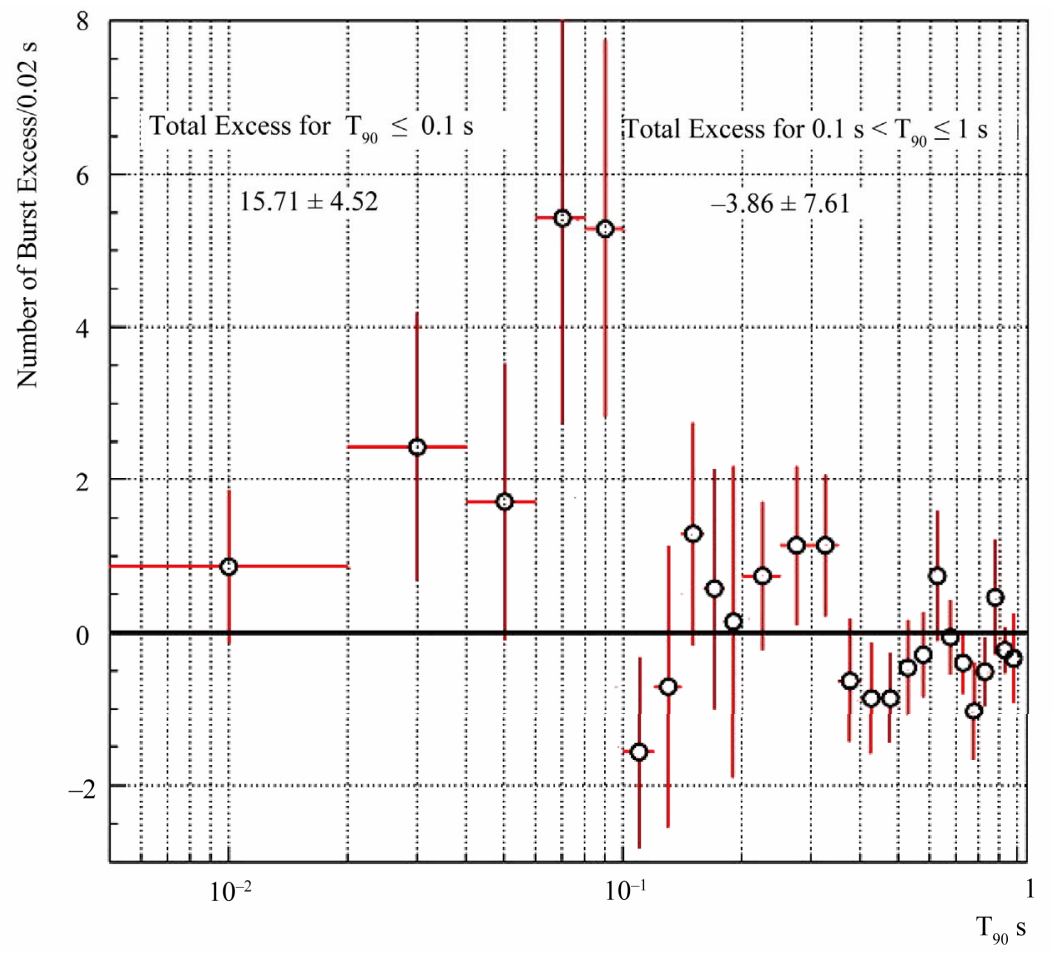

Figure 4. BATSE GRB events (1991 Apr 21-2000 May 26). Excess in GRBs inside the chosen region (see Figure 3) $\left(30^{\circ}<b<\right.$ $-30^{\circ} ; \mathbf{9 0}^{\circ}<l<180^{\circ}$ ) as a function of $\mathrm{T}_{90}[5]$. 


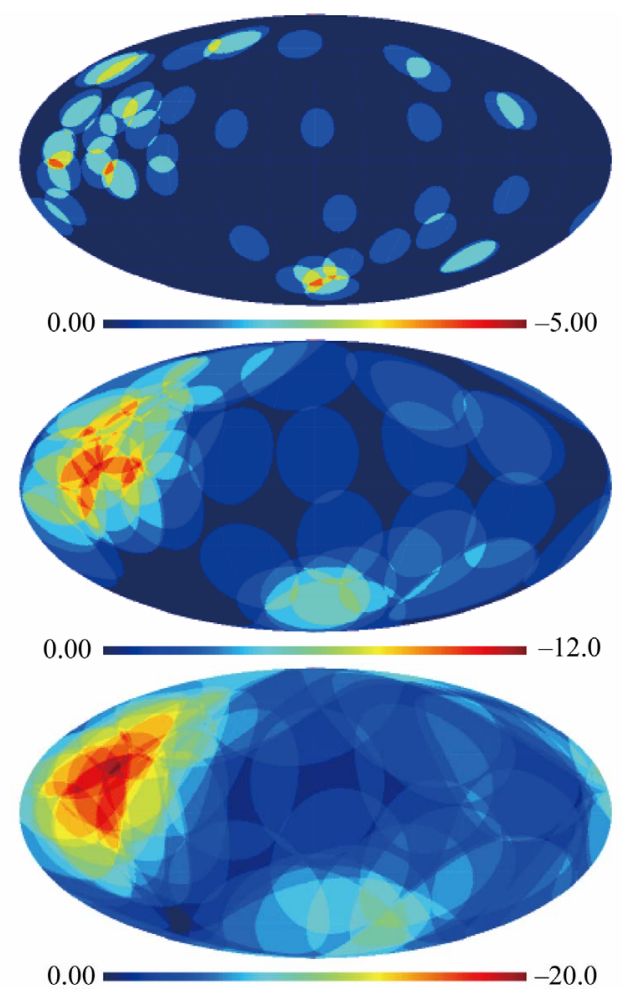

Figure 5. Angular distribution of the BATSE VSGRB events in Galactic Coordinates within $10^{\circ}$ (top), $\mathbf{2 5}^{\circ}$ (middle) and $40^{\circ}$ (bottom) radius cone around each event [6].

order, if there is any structure within this cluster. The result is consistent with the lack of any genuine correlation of $4^{\text {th }}$ and $5^{\text {th }}$ order and suggests that the there are few smaller groups of multiplicity about of $2-3$. This result is "scale-free".

In [7] the possible correlations of VSGRBs with CMB, cosmic rays and particular kind of astronomical objects (Polars) is shown. These correlations show particularity that in Quadrant 2 some astronomical indicators are stronger [7,9]. It is in agreement with our observation of the VSGRB concentration in this region. Is it by chance? We cannot exclude that such concentration of matter was relic from the time of the Big Bang.

\section{V/Vmax Distribution}

We have also reanalyzed the overall radial distribution of the VSGRBs and the SGRB using the standard $\left\langle V / V_{\max }\right\rangle$ test [4]. We used the $C_{\max } / C_{\min }$ table from BATSE catalog as an input to $V / V_{\max }$ calculations: $V / V_{\max }=C_{\max } / C_{\min }=\left(C_{\max } / C_{\min }\right)^{-3 / 2}$. The results are $\left\langle V / V_{\max }\right\rangle=0.52 \pm 0.05$ and $0.36 \pm 0.02$ for VSGRBs and SGRBs respectively [5], see Figure 6. It means that SGRBs appear to come from cosmological distances, but no cosmological effects are seen in the distribution of VSGRBs. We have one more confirmation of the differ- ence between both groups of events.

\section{VSGBBs Have More Energetic $\gamma$-Ray Bursts than Other GRBs with Longer Duration}

In Figure 7 we compare the mean energy of SGRB and VSGRB for KONUS events. Nominal energy range of $\gamma$-ray measurements in KONUS covers the interval from $12 \mathrm{keV}$ up to $10 \mathrm{MeV}$. We observe that in the MeV region the spectra of VSGRB are significantly harder than the spectra of SGRB. The spectrum starts to be flatter above $3 \mathrm{MeV}$ and the effect in the case of VSGRB is stronger [5].

To follow up in Figure 8 we study all SGRB events in KONUS data and select burst with the mean energy $\langle\mathrm{E} \gamma\rangle\rangle$ $90 \mathrm{keV}$. We construct a histogram of burst numbers as a function of their duration. For comparison, we show similar histogram made for SGRs with mean energies $\langle E \gamma\rangle$ $<90 \mathrm{keV}$, and scale it down to the distribution of harder bursts. Comparing these two distributions we see very strong clumping of hard bursts at very short durations. In this histogram in time interval $\mathrm{T}_{90}<0.1 \mathrm{~s}$ we expect one event from softer bursts distribution and found ten hard events, which is extremely unlikely (probability of such fluctuation is $<10^{-10}$ ). That indicates again some new physics origin of the bulk of the VSGRB data [10]. 
BATES GRB EVENTS (SINCE APRIL 21, 1991 TILL MAY 26, 2000)

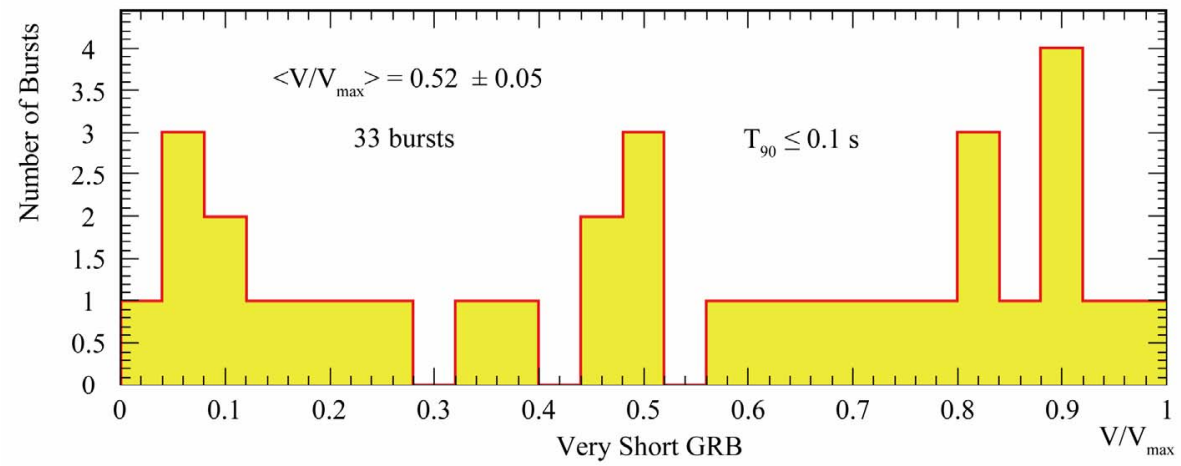

(a)

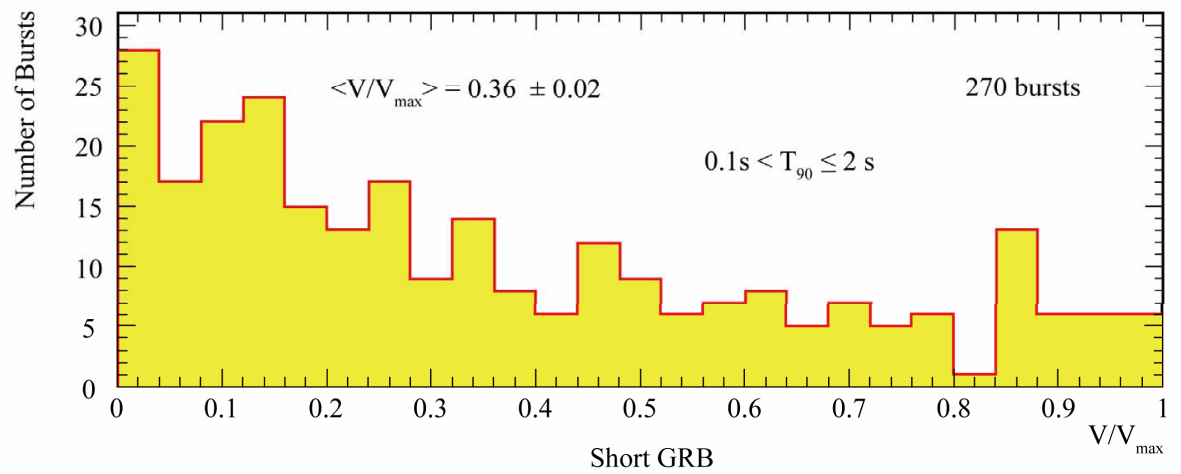

(b)

Figure 6. Distribution of the $V / V_{\max }$ for BATSE events (1991 April 21-2000 May 26) [5].

KONUS GRB EVENTS (SINCE 1995 TILL 2002)

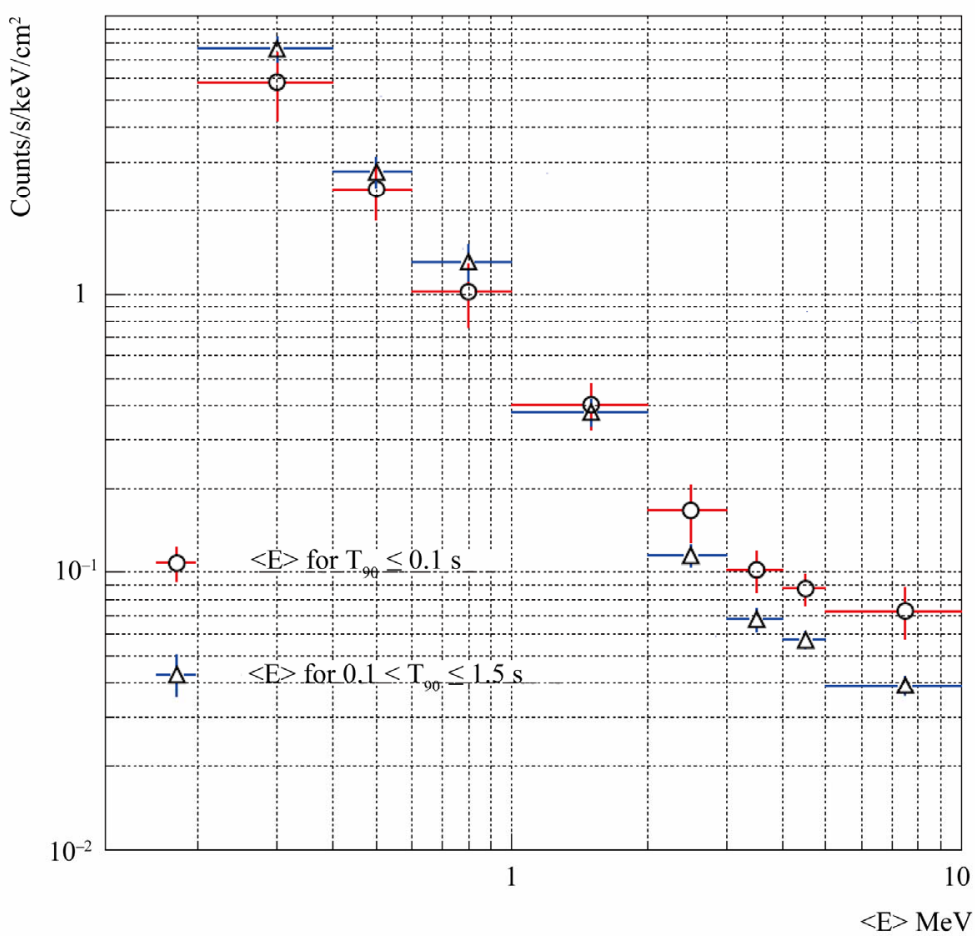

Figure 7. Mean energy distribution for KONUS short and very short bursts [5]. 


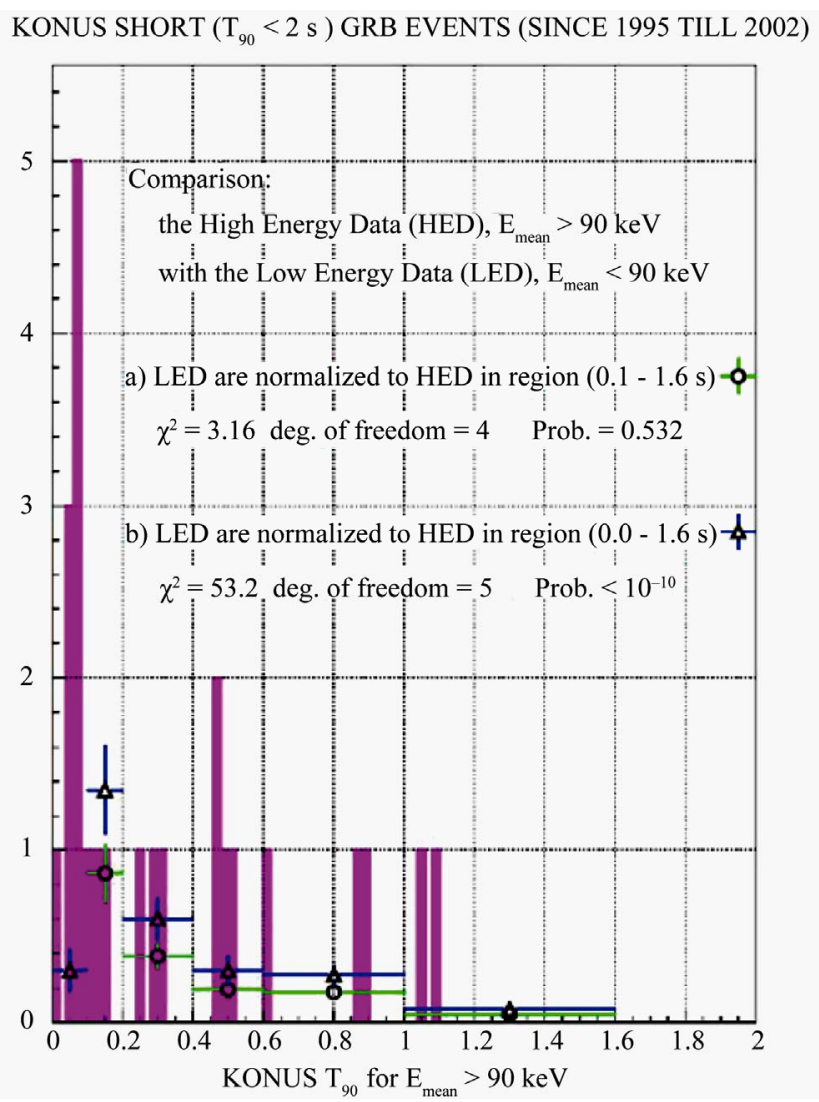

Figure 8. KONUS data with different cuts on the average photon energy $\left\langle E_{\gamma}\right\rangle[10]$.

\section{Afterglows}

In SWIFT VSGRB sample we observe $25 \%$ bursts with afterglows, but in the SGRB sample $\left(0.1<\mathrm{T}_{90}<2 \mathrm{~s}\right)$ we notice $78 \%$ bursts with afterglows. We can interpret it that in VSGRB sample there is about one third of bursts, the tail of the basic SGRB distribution, deriving from the merger of compact objects in binary system (neutron stars -NS or black holes -BH): NS-NS and NS-BH. So two third of VSGRBs are different from SGRBs and can be of new origin, which could be consistent with PBH evaporation (Figure 9).

\section{Time Profile of Rising Part of BATSE VSGRBs is in Agreement with Evaporation PBH.}

For all BATSE VSGRBs the background subtracted lightcurve is normalized by its maximum value and shifted, so the maximum is at $t=0$. The resulting composite profile is shown in Figure 10. The profile indicates significant asymmetry with the timescale of $t_{\text {rise }}=0.00079$ $\mathrm{s}$ for exponential rise and of $t_{\text {fall }}=-0.0171 \mathrm{~s}$ for exponential decay time (Equation 1).

$$
\begin{aligned}
f(t) & =\exp \left(\left(t-t_{0}\right) / t_{\text {rise }}\right) \text { for } t<t_{0} \\
& =\exp \left(-\left(t-t_{0}\right) / t_{\text {fall }}\right) \text { for } t>t_{0}
\end{aligned}
$$

If we use evaporation PBH model, under some assumptions [11], the fit to the rising part of the profile is good. The simple evaporation formula does not give the time profile after the peak; it needs some modifications [11]. Better fit to the decay phase, however, is provided by the function

$$
f(t)=1 /(1+t / \tau)^{n}
$$

with index $n=2.62$ and the decay timescale $\tau=0.0319 \mathrm{~s}$.

If we supposed the phase transition in final state of evaporation-what means "explosion" with upper limit of particles' and $\gamma$ ' energy, then we should expect modification of the final state of Hawking evaporation process. The energy of the phase transition defines the temperature of the fireball, what denominates total mass evaporated. If energy is lower the mass of fireball is bigger.

\section{Conclusions}

- We observe the time $\mathrm{T}_{90}$ distribution of all GRBs detected by 7 experiments and conclude the small surplus 

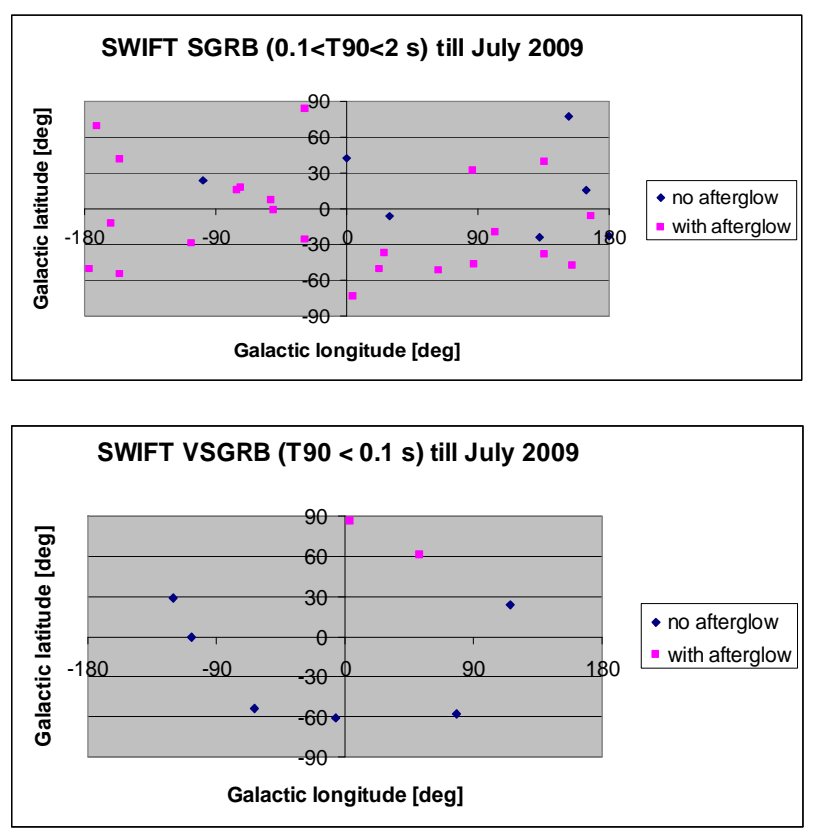

Figure 9. The SWIFT VSGRB and SGRB angular distribution in Galactic coordinates.

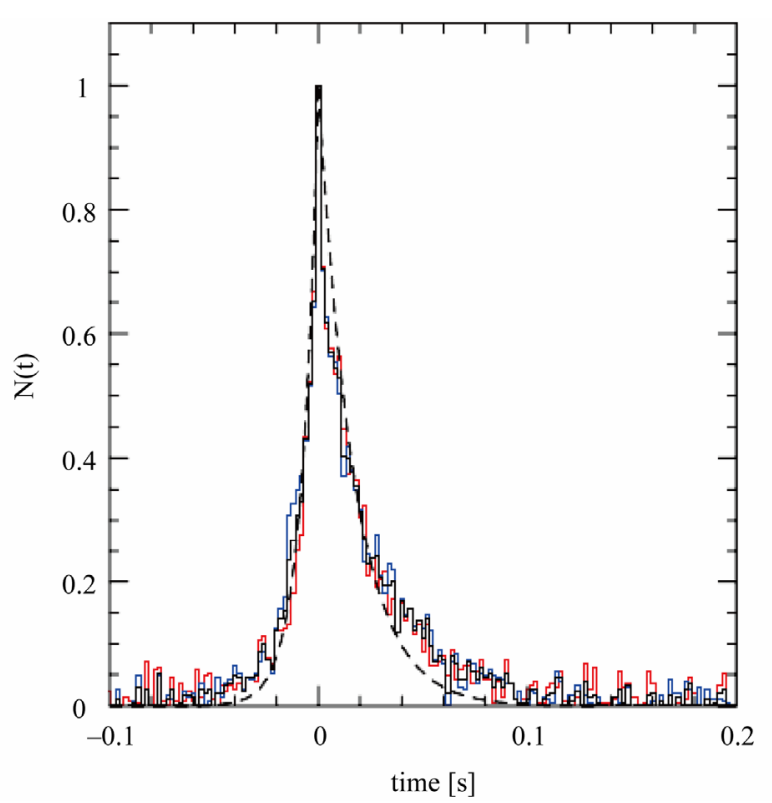

Figure 10. Composite burst profiles for all VSGRB (black line), for bursts from Galactic Anticenter region (red line) and for bursts from outside that region (blue line). The analytical fit (dashed line) is given by Equation 1. Better fit for the decay part is provided by Ryde \& Svensson function (Equation 2) [11].

of VSGRB $\left(\mathrm{T}_{90}<100 \mathrm{~ms}\right)$ in every experiment, meaningful for all together. BATSE VSGRBs coming from Galaxy Anticenter direction show $4 \sigma$ excess for $\mathrm{T}_{90}<$ 0.1 s. Also KONUS data with $\left\langle\mathrm{E}_{\gamma}>>90 \mathrm{keV}\right.$ gives unexpected excess of VSGRBs in comparison with events with $\left\langle\mathrm{E}_{\gamma}><90 \mathrm{keV}\right.$. All these signals suggest treating VSGRB as a new class of events connected with a new origin of the bursts.

- Two independent analyses of BATSE GRB data confirm significant $(4 \sigma)$ anisotropy in the galactic angular distribution of BATSE VSGRB events. It suggests local origin of these VSGRBs when all the others GRBs have cosmological origin. Also V/Vmax distribution for BATSE VSGRB events indicates its local distance production. This confirms also distinct origin of these events.

- VSGBBs have more energetic $\gamma$-ray burst than other GRBs with longer duration (KONUS), see Figure 7 and Figure 8. Such effect we would expect for PBH evaporation. See also Figure 2.

- We observe small number of afterglows in SWIFT VSGRB sample (25\%), in contrast with the noticeable afterglow frequency in SGRB sample (78\%). We can interpret that in VSGRB sample there is about one third of bursts, (the tail of the basic SGRB distribution, deriving from the merger of compact objects in binary system: NS-NS and NS-BH). So two-thirds of VSGRBs are different from SGRBs and can be of new origin, which could be consistent with PBH evaporation.

- The time profile of rising part BATSE VSGRBs is in agreement with evaporation PBH model. We are not sure the theory of PBH evaporation can be applied $[2,4]$. If we supposed the phase transition in final state of evaporation - this provides an "explosion" with upper limit of particles' and $\gamma$ ' energy, then we should expect modification of the final state of Hawking evaporation process. The energy of the phase transition defines the temperature of the fireball, what denominates total evaporated mass. If energy is lower the mass of fireball is bigger.

All these information suggest that VSGRBs create a new class of GRBs and also suggest that these events can be treated in the majority of cases as a result of PBH evaporation ("explosion").

\section{References}

[1] S. W. Hawking, "Black Hole Explosions?" Nature, Vol. 248, 1974, pp. 30-31.

[2] B. J. Carr, et al., "New Cosmological Constraints on Primordial Black Holes," Physical Review D, Vol. 81, No. 10, 2010, arXiv: 0912.5297.

[3] J. B. Carr and S. W. Hawking, "Black Holes in the Early Universe," Monthly Notices of the Royal Astronomical Society, Vol. 168, 1974, pp. 399-416.

[4] D. B. Cline and W. P. Hong, "Possibility of Unique Detection of Primordial Black Hole Gamma-Ray Bursts," Astrophysical Journal, Vol. 401, No. 2, 1992, pp. L57L60. doi:10.1086/186670

[5] D. B. Cline, et al., "Study of Very Short Gamma-Ray 
Bursts: New Results from BATSE and KONUS," The Astrophysical Journal Letters, Vol. 633, No. 2, 2005, p. L73.

[6] L.W. Piotrowski, et al., "Angular Anisotropy of Very Short Gamma Ray Bursts," 2006. http://www.lip.pt/events/2006/ecrs/proc/ecrs06-s5-10.pdf

[7] K. Jedrzejczak, et al., "Search for Correlations of GRB and Cosmic Rays," Astrophysics and Space Science, Vol. 309, No. 1-4, 2007, pp. 471-475. doi:10.1007/s10509-007-9442-7

[8] A. Pozanenko and P. Minaev, "Where is third group of bimodal distribution of T90?".

http://axro.cz/ibws10/media/uploads/pozanenko_v2.pdf
[9] T. Wibig and A. W. Wolfendale, "Foreground Contributions to the Cosmic Microwave Background," Monthly Notices of the Royal Astronomical Society, Vol. 360, No. 1, 2005, pp. 236-252. doi:10.1111/j.1365-2966.2005.09030.x

[10] D. B. Cline, et al., "The Search for Primordial Black Holes Using Very Short Gamma Ray Bursts," 2007, arXiv: 0704.2398v1.

[11] B. Czerny, et al., "Observational Constrains on the Nature of Very Short Gamma-Ray Bursts," New Astronomy, Vol. 16, No. 1, 2011, pp. 33-45. doi:10.1016/i.newast.2010.06.001 